\title{
Application of a Meshed Skin Graft in the Surgical Bed Immediately after Resection of Neurofibrosarcoma in the Distal Limb Region of a Dog
}

\author{
Matheus Cézar Nerone', Rodrigo Casarin Costa², Jorge Luis Alvarez Gómez ${ }^{3}$, Fabiana Del Lama Rocha ${ }^{3}$, \\ Annelise Carla Camplesi ${ }^{4}$ \& Andrigo Barboza de $\mathrm{Nardi}^{4}$
}

\begin{abstract}
Background: Malignant peripheral nerve sheath tumors are neurogenic neoplasms that originate from cells that surround the axons of peripheral nerves. Surgery is the treatment of choice for peripheral nerve sheath tumors. They have a better prognosis when the lesion is in the extremity of a limb and the surgeon leaves wide peripheral margins after resection. However, this procedure makes local treatment a challenge due to difficult wound healing in this region. This report describes a successful case involving the use of a meshed skin graft immediately after resection of a neurofibrosarcoma in the distal region of the radius bone of a dog.

Case: A 6-year-old Boxer bitch weighing $40 \mathrm{~kg}$ was admitted with a history of a round, firm, non-ulcerated skin nodule attached to the lateral side of the distal region of the right radius bone, which had been present for about 40 days. Fine needle aspiration cytology of the lesion showed the presence of mesenchymal cells, suggesting a sarcoma. Thus, the decision was made for an incisional biopsy to confirm the diagnosis and for the preparation of a subdermal pattern tubular flap for subsequent rotation and transposition to close the wound that would be formed after the complete removal of the lesion. After the $7^{\text {th }}$ postoperative day, the diagnosis of low-grade neurofibrosarcoma was confirmed and due to the presence of necrotic onset in the middle portion of the tubular flap, further surgical intervention was scheduled for the resection of the tube flap, en bloc removal of the neoplastic lesion with peripheral margins of $2 \mathrm{~cm}$, and wound closure with a free skin graft. A mesh skin graft was made with a portion of the right flank skin. The mesh graft was carefully implanted on the recipient bed using simple interrupted sutures with a 3-0 non-absorbable monofilament suture material. A dressing made with water-based sterile lubricating solution and gauze and a modified Robert Jones bandage were applied. The patient was treated with analgesic medication, antimicrobial therapy, and gastric protectors. The first bandage and dressing were changed after $48 \mathrm{~h}$, and only warm physiological solution was used. Histopathological analysis confirmed the diagnosis of low-grade neurofibrosarcoma with free surgical margins. The dressing was changed every $72 \mathrm{~h}$ for up to 12 postoperative days, when the immobilization was removed, and wound cleaning could be completed at home using physiological solution and a dressing with nitrofurazone ointment. Granulation tissue was first seen at day 12, and at day 30 the wound was completely closed.

Discussion: The primary closure of skin defects after the resection of tumors located in the distal portion of limbs is often not possible due to a lack of adjacent skin. Thus, grafts are commonly used to repair the skin in these areas using the technique presented in this report. In order to survive, skin grafts need a vascular bed capable of allowing new blood vessel connections with the implanted skin portion. There is no consensus in the literature on the best bed for graft implantations. In the case described in this study, a free mesh graft was implanted on a fresh wound without granulation tissue, which resulted in excellent clinical evolution and total survival of the graft. Excellent clinical results were achieved with the graft, since its survival and adherence to the recipient bed occurred without complications. The clinical result of this case suggests that the application of full-thickness mesh grafts to fresh wounds in distal limbs immediately after tumor resection is a good alternative when the surgical wound is too large (which prevents primary closure). Good preoperative planning associated with good surgical techniques and adequate postoperative management are essential for the success of the technique under these conditions.
\end{abstract}

Keywords: graft, canine, neoplasia, reconstructive surgery. 
M.C. Nerone, R.C. Costa, J.L.A. Gómez, et. al. 2020. Application of a Meshed Skin Graft in the Surgical Bed Immediately after Resection of Neurofibrosarcoma in the Distal Limb Region of a Dog. Acta Scientiae Veterinariae. 48(Suppl 1): 497.

\section{INTRODUCTION}

Malignant peripheral nerve sheath tumors (MPNSTs) are neurogenic neoplasms that originate from cells that surround the axons of peripheral nerves. Although they are locally invasive, pulmonary metastasis is rare [4]. The prognosis is poor, with high rates of relapse [6]. In contrast, MPNSTs located in distal nerves have a more favorable prognosis [4].

Surgery is the treatment of choice for peripheral nerve sheath tumors [4]. Many techniques have been described for limb wound healing, such as axial pattern flaps, tube flaps, tissue expanders, microvascular free tissue transfer, and grafts [10]. The success of a technique varies depending on the location of the lesion, since the total removal of the affected tissue with wide peripheral margins will lessen the probability of relapse $[4,6]$. Thus, although MPNSTs in the extremities of limbs have a better prognosis [4], their local treatment becomes a challenge due to the small amount of loose skin in the region, greater mobility, and greater distance from possible donation areas, which make wound healing through skin mobilization or flap reconstruction more difficult $[3,11]$.

There is no consensus in the literature on the best time for a free skin graft implantation, either on a fresh wound or after granulation of the wound bed, so the objective of this report is to describe a successful outcome following the local resection of a neurofibrosarcoma and immediate grafting of the surgical wound in the distal region of the radius bone of a dog.

\section{CASE}

A 6-year-old Boxer bitch weighing $40 \mathrm{~kg}$ was admitted with a history of a round, firm, nonulcerated skin nodule attached to the lateral side of the distal region of the right radius bone, which had been present for about 40 days. Hematological tests, chest radiographs in the right and left ventrodorsal and laterolateral projections, and abdominal ultrasonography showed no abnormalities. Fine needle aspiration cytology of the lesion showed the presence of mesenchymal cells, suggesting a sarcoma.

Therefore, the decision was made for an incisional biopsy to confirm the diagnosis and for the preparation of a subdermal pattern tubular flap for subsequent rotation and transposition to close the wound that would be formed after the complete removal of the lesion. The latter would require a second surgical procedure.
After the $7^{\text {th }}$ postoperative day, the diagnosis of low-grade neurofibrosarcoma was confirmed and due to the presence of necrotic onset in the middle portion of the tubular flap, further surgical intervention was scheduled for the resection of the tube flap, en bloc removal of the neoplastic lesion with peripheral margins of $2 \mathrm{~cm}$, and wound closure with a free skin graft.

During surgery, the patient was placed in the left lateral decubitus position and after the entire right thoracic limb, abdomen, and right flank were shaved, the peripheral margins were marked, and the area was anti-septicized (Figure 1A). A surgical team removed the tube flap, while another team removed the neoplastic lesion en bloc, while also removing the fascia and exposing the digital tendons (Figure 1B). After changing the surgical materials and dressings, the recipient bed was washed with physiological solution and the defect was protected with a dressing moistened with sterile saline solution.

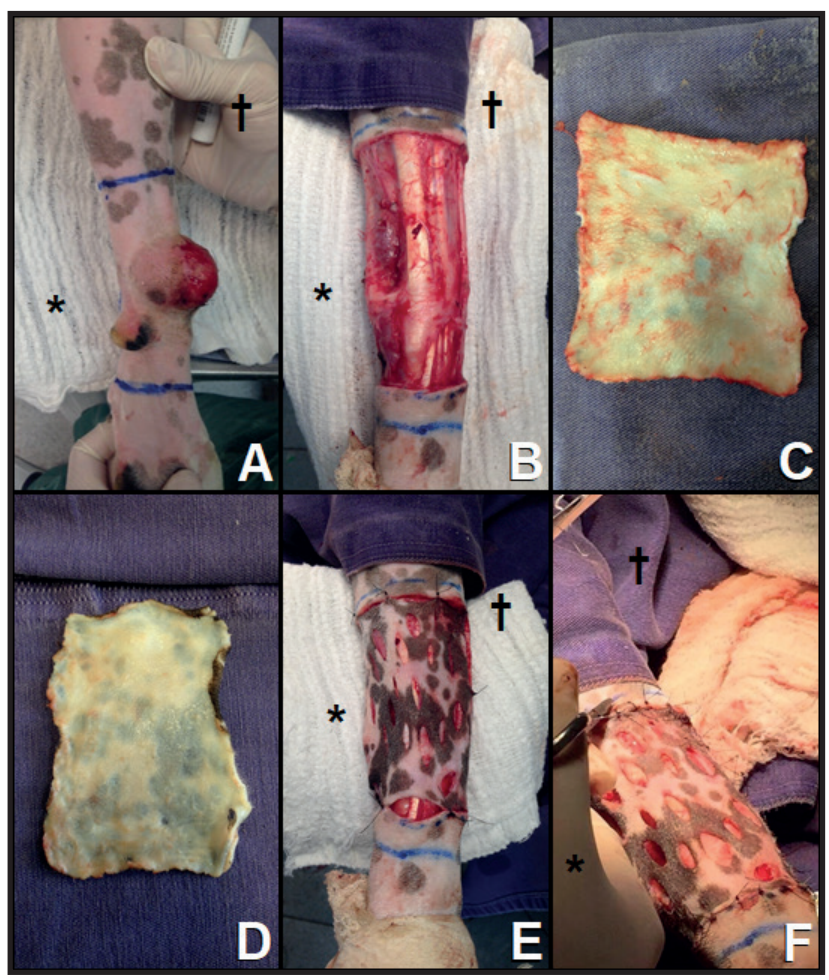

Figure 1. Perioperative photographic images of the neurofibrosarcoma resection on the lateral distal radius bone of the right thoracic limb and of the lesion closure with a free mesh graft. The black asterisk indicates the caudal portion and the black cross indicates the proximal portion of the limb. A- Surgical planning with delineation of surgical margins and en bloc removal of the neurofibrosarcoma (blue arrow), including the carpal pad (black arrow). B- Final appearance of the surgical wound after en bloc removal of the lesion with exposure of the muscles and digital tendons. C- Skin graft harvested from the left flank with presence of subcutaneous tissue remains. D- Skin graft after removal of the subcutaneous tissue remains. E- Beginning graft implantation (white arrow) in the recipient bed using simple interrupted stitches with a non-absorbable monofilament suture (yellow arrow). F- Final aspect of the mesh grafting. 
A portion of the right flank skin with the same proportions of the recipient bed defect had been carefully harvested with the use of a scalpel, atraumatic forceps, and surgical scissors (Figure 1C). After the complete removal of subcutaneous tissue from this skin portion, longitudinal incisions were made on it to characterize a mesh skin graft. The mesh graft was carefully implanted on the recipient bed using simple interrupted sutures with a 3-0 non-absorbable monofilament suture material (Figure 1D, 1E, \& 1F).

After the closure of the donor bed defect, a dressing made with water-based sterile lubricating solution and gauze was applied. Due to the hyperactive behavior of the patient and the location of the graft, which was close to the joint, a modified Robert Jones bandage was also used.

The patient was treated with analgesic medication, antimicrobial therapy, and gastric protectors. The first bandage and dressing were changed after 48 $\mathrm{h}$, and only warm physiological solution was used. The dressing and bandage were then re-applied as previously described (Figure 2A). Histopathological analysis confirmed the diagnosis of low-grade neurofibrosarcoma with free surgical margins.

The dressing was changed as described above every $72 \mathrm{~h}$ for up to 12 postoperative days, when the immobilization was removed, and wound cleaning could be completed at home using physiological solution and a dressing with nitrofurazone ointment $\left(\text { Furacin }^{\circledR}\right)^{1}$.

During the first eight postoperative days, the graft presented areas of hematoma at the extremities, but without signs of necrosis (Figure 2B). Granulation tissue was first seen at day 12 , and at day 30 the wound was completely closed (Figure 2C \& 2D). Thus, the patient was discharged with a recommendation to avoid sun exposure because the animal had light-colored skin and the grafted region had no hair (Figure 2E).

\section{DISCUSSION}

The primary closure of skin defects after the resection of tumors located in the distal portion of limbs is often not possible due to a lack of adjacent skin [8-10], especially when it is a large tumor with wide surgical margins [10]. Thus, grafts are commonly used to repair the skin in these areas using the technique presented in this report [8].

In the case reported here, a subdermal pattern tubular flap was created prior to tumor resection to be used in a second surgery, when the rotation and transposition of the flap would be used to close the surgical wound after the complete removal of the tumor. However, the onset of necrosis was observed in the middle portion of the flap one week after the tube was made. The authors of this study believe that this happened because it was a subdermal pattern tube and was too long, thus reducing the blood supply in the middle portion of the tube and leading to local ischemia and consequent necrosis of the region, as described by Macomber \& Rubin [5], who mentioned that the length of the tube should be balanced with an adequate width to ensure adequate blood supply in its center.

In order to survive, skin grafts need a vascular bed capable of allowing new blood vessel connections with the implanted skin portion [1]. Grafts applied on hypertrophic granulation tissues will not survive, since the recipient bed tissue does not have adequate vascularization to maintain the graft [3]. There is no consensus in the literature on the best bed for graft implantations. In the case described in this study, a free mesh graft was implanted on a fresh wound without granulation tissue, which resulted in excellent clinical evolution and total survival of the graft,

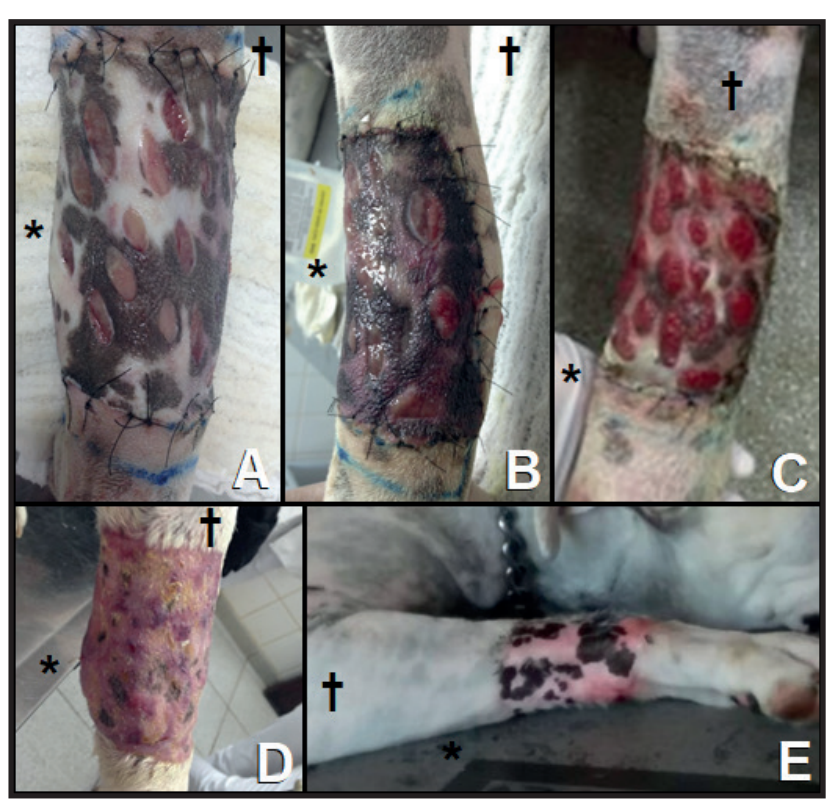

Figure 2. Postoperative photographic images after the removal of the neurofibrosarcoma on the lateral distal radius bone of the right thoracic limb and lesion closure with a free mesh graft. The black asterisk indicates the caudal portion and the black cross indicates the proximal portion of the limb. A- $48 \mathrm{~h}$ after surgery, an initial hematoma at the extremities of the graft can be seen (red arrow). B- 5 days after surgery, showing the worsening of the hematoma in the extremities (red arrow), but with no signs of necrosis. C- 12 days after surgery, granulation tissue is evident in the graft fenestrations (white arrow). D- 30 days after surgery showing a completely closed wound (yellow arrow). E- Final postoperative appearance, showing the graft area with no hair growth (black arrow). 
which is in disagreement with Pazzini et al. [7], who recommended the use of a graft at a later date, after granulation tissue had formed on the bed. However, this corroborates the account given by Bohling and Swaim [3], who discussed the possibility of graft application on a fresh receptor bed. In addition, some studies suggest that faster vascularization may occur in grafts placed on fresh tissue than in grafts placed on granulated tissue [3].

Tong \& Simpson [10] evaluated the applicability of full-thickness mesh skin grafts on fresh surgical wounds after excision of tumors located in the distal portion of the limbs. In this study, neoplastic resection was performed in the forearm of six dogs and in the tarsal metatarsal region of one dog. As in the present case report, the recipient beds were composed mainly of adjacent musculature and tendons. Three grafts survived completely and partial and superficial epidermal necrosis occurred in some areas in the remaining four grafts. Of the three cases that did not present with any type of necrosis, similar to our case, two used the left flank as the donor bed, which was not the graft harvest site for any of the cases in which necrosis occurred. This may be an indication that the region from which the graft was harvested may have an influence on the optimal results we achieved.

Tong and Simpson [10] reported on a postoperative care procedure similar to the one used in this report. We believe that the high rate of epidermal necrosis occurred due to the location of the grafts in the caudal area of the affected distal forearm. It may be related to the irregular surface and difficulties in completely eliminating the movement of the tendons at the site of the graft bed.

There was no hair regrowth in the grafted region in this present study, probably due to possible involuntary damage of hair follicles in the deep dermis during graft preparation. Other factors that may have impacted on hair regrowth in the graft area include epidermal follicular necrosis, apocrine gland damage, or revascularization problems during engraftment [2]. Nevertheless, excellent clinical results were achieved with the graft, since its survival and adherence to the recipient bed occurred without complications. In contrast, the cosmetic result was worse than expected due to the absence of hair regrowth in the region.
The success rate of skin grafts for repairing wounds on extremities seems to be significantly higher in cats than dogs [8,9]. Riggs et al. [8] conducted a study to evaluate the use of full-thickness skin grafts to close skin defects in the distal areas of limbs in both cats and dogs. There were successful results in 17 of the $22(77 \%)$ grafts implanted in cats and in 12 of the $32(38 \%)$ grafts implanted in dogs. In the same study, the most common graft receptor site was the metatarsal region, followed by the tarsal region, the metacarpal region, the forearm, the carpal region, and then the elbow. In another study, 17 full-thickness skin grafts were used to treat extensive skin defects in the distal limbs of 16 cats, with a success rate of $82 \%$ [9]. The authors attributed the high success rate in this series of cases to adequate preparation of the recipient bed, graft harvest, and postoperative bandaging. However, unlike our case, the graft recipient bed of their study was formed by granulation tissue [9].

The use of adequate postoperative bandaging is essential for the success of a skin graft [9]. The bandage change interval can range from once a day to once every two or four days. The first postoperative bandage is usually left intact for 24 to $48 \mathrm{~h}[3,8]$ to reduce the occurrence of seromas or hematomas that can present at this stage, thus ensuring the contact between the graft and the recipient bed and, consequently, graft viability [8].

The main limitation of the present study is that it was based on only one clinical case. Further research, using a larger animal population, is necessary to corroborate the results obtained in this study. The clinical result of this case suggests that the application of full-thickness mesh grafts to fresh wounds in distal limbs immediately after tumor resection is a good alternative when the surgical wound is too large (which prevents primary closure). Good preoperative planning associated with good surgical techniques and adequate postoperative management are essential for the success of the technique under these conditions.

\section{MANUFACTURER}

${ }^{1}$ Mantecorp Indústria Química e Farmacêutica Ltda. Rio de Janeiro, RJ, Brazil.

Declaration of interest. The authors report no conflicts of interest. The authors alone are responsible for the contents and writing of the paper. 


\section{REFERENCES}

1 Amsellem P. 2011. Complications of reconstructive surgery in companion animals. Veterinary Clinics of North America: Small Animal Practice. 41(5): 995-1006.

2 Bhandal J., Langorh I.M., Degner D.A., Xie Y., Stanley B.J. \& Walshaw R. 2012. Histomorphometric Analysis and Regional Variations of Full Thickness Skin Grafts in Dogs. Veterinary Surgery. 41(4): 448-454.

3 Bohling M.W. \& Swaim S.F. 2017. Skin grafts. In: Johnston S.A. \& Tobias K.M. (Eds). Veterinary Surgery Small Animal. 2nd edn. St. Louis: Elsevier Saunders, pp.3951-4001.

4 Gibson A.D., Davies E., Lara-Garcia A. \& Lafuente P. 2016. Palliative Epineurotomy for Focal Radial Malignant Peripheral Nerve Sheath Tumor in a Dog. Journal of the American Animal Hospital Association. 52(5): 330-334.

5 Macomber W.B. \& Rubin L.R. 1947. Tubed pedicle complications in repair of massive tissue defects. Plastic and Reconstructive Surgery. 2(1): 10-20.

6 Patterson C.C., Perry R.L. \& Steficek B. 2008. Malignant peripheral nerve sheath tumor of the diaphragm in a dog. Journal of the American Animal Hospital Association. 44: 36-40.

7 Pazzini J.M., De Nardi A.B., Castro J.L.C. \& Huppes R.R. 2016. Cirurgia reconstrutiva aplicada na oncologia. In: Daleck C.R. \& De Nardi A.B. (Eds). Oncologia em Cães e Gatos. 2.ed. Rio de Janeiro: Editora Roca Ltda., pp.179-186.

8 Riggs J., Jennings J.L.F., Friend E.J., Halfacree Z., Nelissen P., Holmes M.A. \& Demetriou J.L. 2015. Outcome of full-thickness skin grafts used to close skin defects involving the distal aspects of the limbs in cats and dogs: 52 cases (2005-2012). Journal of the American Veterinary Medical Association. 24(9): 1042-1047.

9 Shahar R., Shamir M.H., Brehm D.M. \& Johnston D.E. 1999. Free skin grafting for treatment of distal limb skin defects in cats. The Journal of Small Animal Practice. 40(8): 378-382.

10 Tong T. \& Simpson D.J. 2012. Free skin grafts for immediate wound coverage following tumour resection from the canine distal limb. Journal of Small Animal Practice. 53(9): 520-525.

11 Tsioli V. \& Dermisiadou E. 2016. Management of distal limb skin defects in dogs and cats. Journal of the Hellenic Veterinary Medical Society. 67(1): 5-16. 